r ScIDioc

\section{International Journal of Dentistry and Oral Science (IJDOS) ISSN: 2377-8075}

\title{
The Camouflage Effect
}

Research Article

\section{Niharika Kotian ${ }^{1 *}$, Vignesh Ravindran ${ }^{2}$}

${ }^{1}$ Post-Graduate Student, Department Of Pedodontics, Saveetha Dental College and Hospitals, Saveetha Institute of Medical and Technical Sciences, Saveetha University, Chennai, India.

${ }^{2}$ Senior Lecturer, Department Of Pedodontics, Saveetha Dental College and Hospitals, Saveetha Institute of Medical and Technical Sciences, Saveetha University, Chennai, India.

\section{Abstract}

Background: Pediatric dentistry is different than dentistry practiced in adults. Children tend to be more anxious before a dental treatments which prevent them from cooperating whole heartedly before any procedure. This is also one of the most basic reasons for avoidance and neglect of dental care. Dental setting plays a very important role in managing a child's anxiety. Making the atmosphere around the child positive by adding the child's favourite color in the dental set up will definitely contribute in improving the anxiety of the child prior a treatment.

Aim: To analyse the effect of colors on children's emotions and variations in anxiety levels using different colored dental operatory equipment and instruments.

Objective: This study is done to evaluate the association between colors and emotions in pediatric dental practice.

Material and Methods: 50 anxious children who were classified based on modified child dental anxiety scale were included in the study. Children were asked to choose a color to shade the happy face drawn on a piece of paper given to them. The dental operatory equipment and armamentarium was covered in the color chosen by the child and then the procedure was performed. The anxiety level in the child was assessed again.

Results: There was significant difference in the anxiety of the child before and after the treatment. Yellow was the most chosen color to depict positive emotion in the child.

Conclusion: The use of child friendly colors in the workplace like yellow and blue could enhance a positive dental attitude in a child's mind.

Keywords: Color; Pediatric Dentist; Anxiety; Emotion.

\section{Introduction}

The child's anxiety is a major concern for all pediatric dentists [1]. Anxiety prior to any dental procedure amongst children of various age groups is recognized to be a major public health dilemma [2]. Children tend not to cooperate during dental procedures, which makes it difficult for the pediatric dentists to manage while performing dental procedures. Pediatric patients tend to become more anxious on seeing the dental armamentarium around them. Colors that induce positive feelings tend to reduce anxiety. A colorful work set up around the child will improve his/ her attitude towards the treatment [3]. In 1939, Goldstein stated that certain colours elicit certain emotional responses. It can be a stress relieving factor to all [4].

Colors perceived by child have a positive or negative effect on their emotions as they link a particular color to a particular emotion [5]. It has been discovered that colors have a role to play in a child's conduct in dental set up [6]. Most of the existing research have been focused on the primary care of adults and these results

\section{*Corresponding Author:}

Niharika Kotian,

Post-Graduate Student, Department Of Pedodontics, Saveetha Dental College and Hospitals, Saveetha Institute of Medical and Technical Sciences, Saveetha University, Chennai, India.

Tel: 8879787839

Email Id: niharikagkotian@gmail.com

Received: April 18, 2021

Accepted: May 28, 2021

Published: May 30, 2021

Citation: Niharika Kotian, Vignesh Ravindran. The Camouflage Effect. Int J Dentistry Oral Sci. 2021;08(05):2554-2557. doi: http://dx.doi.org/10.19070/2377-8075-21000500

Copyright: Niharika Kotian ${ }^{\circ} 2021$. This is an open-access article distributed under the terms of the Creative Commons Attribution License, which permits unrestricted use, distribution and reproduction in any medium, provided the original author and source are credited. 
cannot be extrapolated for a child. A study conducted by Park et al was done to investigate the value of color as a component of a healing environment for pediatric patient room [7]. The authors concluded that, yellow was the preferred color by pediatric patients to express their emotion.

There have been many studies performed on color but hardly any studies performed on correlation between a child's emotion and the color of the armamentarium used in a dental set up. The purpose of the current study was to assess the child's anxiety level in undergoing the dental procedure in a colored dental setup of their choice.

\section{Materials and Methods}

The present study was conducted in accordance with the guidelines given by the CONSORT checklist. The present study was conducted in the department of Pediatric \& Preventive Dentistry in a dental institute, Chennai. The study design was reviewed and approved by the institutional review board. The research proto- cols and methods were informed to the parent/care-giver and an informed consent was obtained from them prior the procedure.

Sample size calculation was determined from a previous study by Jayakaran et al [2] using G-Power with $85 \%$ power. A total of fifty children between 3 to 6 years of age were included for the study. Inclusion criteria for the study were children between 3-6 years of age, anxious nature prior to treatment and children requiring preventive therapy and restorations. Exclusion criteria were children with cooperative for dental treatment, children who needed emergency dental treatments, children with systemic conditions and children who require treatments involving local anesthesia.

A specially prepared proforma was used to record personal information which included Modified Child's Dental Anxiety Scale given by Wong et al in 1998. Modified child dental anxiety scale has a 5-point Likert scale with the values 1 [Relaxed / not worried], 2 [Very slightly worried], 3 [Fairly worried], 4 [Worried a lot] and 5 [Very worried]. These children were assessed based on the anxiety scale at the receptionist area itself. During their waiting

Figure 1. Children coloring smiley face with color of their choice.

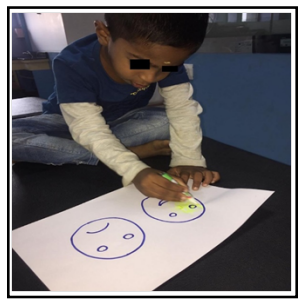

Figure 2. Armamentarium, dental chair and mask of pediatric dentist in the color chosen by the child.

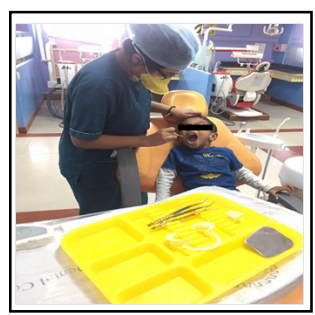

Figure 3. Choice of colors by children depicting positive emotion.

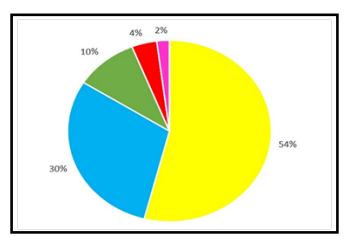

Table 1. Summary of demographic variables describing sample size and number of males and females participants.

\begin{tabular}{|c|c|c|}
\hline Total & Males & Females \\
\hline 50 & 22 & 23 \\
\hline
\end{tabular}

Table 2. Anxiety level prior and during the dental procedure.

\begin{tabular}{|c|c|c|c|c|}
\hline & N & Mean & Standard deviation & P value \\
\cline { 1 - 3 } Anxiety scale rating before treatment & \multirow{2}{*}{50} & 3.0 & 0.28 & \multirow{2}{*}{0.000} \\
\cline { 1 - 1 } Anxiety scale rating after treatment & & 1.24 & 0.43 & \\
\hline \multicolumn{4}{|c|}{$\mathrm{p}<0.05$ was considered statistically significant }
\end{tabular}


period, the children were given an activity. The activity was to color on a plain and bright white paper which had a smiley face with black outline drawn on it. The children were also given 6 crayons of different colors. The colors included were yellow, blue, green, red, pink and black. The children were asked to color the smiley face with a color of their choice [Figure 1]. Once the child had completed coloring the picture, the color was assessed by the receptionist. The receptionist informed the dental assistant about the color used for the picture. The dental assistant was asked to cover all the armamentarium required for the dental procedure in that particular color using colored cellophane sheets. The face mask and the surgical glove used by the pediatric dentist (who would be performing the dental treatment) were also chosen in the same color of choice [Figure 2]. The child was accompanied by the same pediatric dentist to the dental chair with the color of his/her choice. Dental chairs were available in all the six colors chosen for the study. The dental treatment was performed by the same pediatric dentist for all the subjects. The dental treatments involved were treatments which did not have a painful stimuli like using a local anesthesia. The treatments that were performed in the study were scaling, pit and fissure sealant application, fluoride varnish application and class I GIC restorations. The anxiety level of the child was recorded during the procedure using the same scale by another investigator (undergraduate student) who had no information about the study protocol. The values were tabulated and subjected to statistical analysis. Chi-square test (SPSS version 15) was used to evaluate the association between the variables and $\mathrm{P} \leq 0.05$ was considered statistically significant.

\section{Results}

Out of 50 children, 22 were boys and 23 were girls (Table 1 ). Out of all the children, 27 children chose yellow to color the smiley face. 15 children chose blue, 5 chose green, 2 chose red and one chose pink and none chose black [Figure 3]. The mean anxiety rating scale for all the children before the treatment was 3 and after the treatment was 1.24 (Table 2). It was observed that anxiety in children during the treatment was improved as compared to the anxiety prior the treatment, which was statistically significant $(\mathrm{p}<0.05)$.

\section{Discussion}

Visiting the dentist can induce emotions of strong anxiety in many people, especially children [8]. Colors form the major part in a child's life. A child's environment including his/her clothes, toys, and home accessories convey many psychological messages through colors [9]. Goldstein claimed that specific color elicits specific emotional responses [10]. Goethe in 1840 created a color wheel showing the psychological effect of each color [11]. So this study was planned to identify the color of the armamentarium that the child would expect to have for reducing their fear/anxiety and there by getting a positive dental attitude. The results showed that children do have preferential colors and can match these colors to emotions. Specific colors and combinations can psychologically affect the vast majority of people regardless of their culture or past [1].

An activity was provided in the initial visit during the waiting period. This was done to ease the child with the dental environment and also to assess their color of preference. Crayon colors were preferred rather than sketch colors as the glossy finish of the crayons tend to create a more satisfactory color hue. The activity paper had only black and white colors on it and with no pre-colored images to avoid the dull shades of color of printing them in the paper, which would eventually suppress the purpose of color emotions. This also tends not to influence the child towards a particular shade of color and to make the choice mostly self-driven.

The bond between color and emotion preferences varied with age i.e. as the individual gets older, their preferences change based on their experiences [5]. So the younger age groups had a better correlation between colors and emotions involved, when compared to the older age groups. This was one of the reasons for recruiting children between 3-6 years of age for the current study. The other reason was to color train the child during the initial stages of the dental visit which would make them associate it to positive behaviours during dental treatments.

Six identifiable colors were selected in this study namely yellow, blue, green, red, pink and black. The four primary colors from the Munsell system were yellow, blue, green and red [12]. Pink was also one of the colors which children tend to relate to especially girls and an achromatic color black was selected. Ekman and Friesen defined six emotions that included happiness, surprise, anger, sadness, aversion, and fear [13]. Terwogt and Hoeksma have shown that children and adults ranked their emotions differently [5].

It has been found that the associations of some mood tones with particular colors are more apparent and precise than others. Yellow is associated with happiness and a positive emotional state; Blue is associated with security, calmness, and comfort; Green with quietness; Red with anger, aggression, and excitation; and Black with depression or anxiety [1]. Cimbalo et al concluded that children used yellow, blue, green and orange to color while viewing happy scenes [14]. Odom et al concluded that yellow was associated with a cheerful and exciting emotion and blue was associated with calmness [15].

The result of this study was that yellow was the most preferred color for positive emotion, followed by blue, green, red, pink. Black was not chosen by any child. It was found that there was significant reduction in their anxiety while the child was under going the dental procedure. This is possibly due to an instilled positive emotion in the child after they saw all the dental operatory equipment in their favourite color and also the operator wearing masks and gloves of their favourite color. This led to a more calming effect while undergoing the procedure as the children developed a sense of trust in the operator, thinking that the operator considered their emotion important and took an effort to make things easier for them while the undergo the treatment. Colors of the plus side of Goethe's color wheel (gradient of red to yellow) produce excitement and cheerfulness. Colors of the minus side of Goethe's color wheel (gradient of green to blue) were associated with weakness and unsettled feelings. The complementary color scheme consists of two colors that are opposite to each other on the color wheel. This scheme looks best when you place a warm color against a cool color. This suggests that yellow can be the primary color and color blue can be an adjunct to it in the dental environment [1]. This correlated well with the results of the current study. 
This study has attempted to advance the area of color research to dental environment. It has also attempted to correlate the preference of color to the emotions of children in a dental set-up. Emotions are not the same for every individual and emotions triggered by a color usually depends on previous experiences, preferences and memories associated with that particular color. One of the limitations of the study can be the smaller sample size. Future studies with larger sample size and larger variation in colors could help establish a strong correlation between different colors and emotion and help pediatric dentists to manage the anxiety levels of their patients. Minor treatments without involving a painful stimuli like scaling, restorative management were considered for the study. This could be one of the reasons for obtaining a more positive behaviour in the current study. Including children with treatments necessitating a use of local anesthesia would provide a better insight on the actual link between the color emotions and the behaviour of the child.

\section{Conclusion}

There was significant difference in the anxiety levels before the treatment and after the treatment. Yellow and blue were the most preferred colors by the child to depict their positive emotion. The use of child friendly colors in the workplace could enhance a positive dental attitude in a child's mind.

\section{References}

[1]. Umamaheshwari N, Asokan S, Kumaran TS. Child friendly colors in a pediatric dental practice. J Indian Soc Pedod Prev Dent. 2013 OctDec;31(4):225-8. Pubmed PMID: 24262394.

[2]. Jayakaran TG, Rekha CV, Annamalai S, Baghkomeh PN, Sharmin DD. Preferences and choices of a child concerning the environment in a pediatric dental operatory. Dent Res J (Isfahan). 2017 May-Jun;14(3):183-187. Pubmed PMID: 28702059.
[3]. Debs NN, Aboujaoude S. Effectiveness of Intellectual Distraction on Gagging and Anxiety Management in Children: A Prospective Clinical Study. J Int Soc Prev Community Dent. 2017 Nov-Dec;7(6):315-320. Pubmed PMID: 29387614

[4]. Rayen R, Muthu MS, Chandrasekhar Rao R, Sivakumar N. Evaluation of physiological and behavioral measures in relation to dental anxiety during sequential dental visits in children. Indian J Dent Res. 2006 JanMar;17(1):27-34. Pubmed PMID: 16900892.

[5]. Terwogt MM, Hoeksma JB. Colors and emotions: preferences and combinations. J Gen Psychol. 1995 Jan;122(1):5-17. Pubmed PMID: 7714504

[6]. Boyatzis CJ, Varghese R. Children's emotional associations with colors. J Genet Psychol. 1994 Mar;155(1):77-85. Pubmed PMID: 8021626.

[7]. Park JG. Color perception in pediatric patient room design: healthy children vs. pediatric patients. HERD. 2009 Spring;2(3):6-28. Pubmed PMID: 21165833.

[8]. Hotwani K, Sharma K. Assessment of the impact of colors on child's anxiety and treatment preference for local anesthesia injections. Journal of Advanced Oral Research. 2017 May;8(1-2):42-6.

[9]. Birren F. Color \& Human Response: Aspects of Light and Color Bearing on the Reactions of Living Things and the Welfare of Human Beings. John Wiley \& Sons; 1984. p176.

[10]. Goldstein K. Some experimental observations concerning the influence of colors on the function of the organism. Occupational Therapy. 1942.

[11]. Goethe. Effect of colors with reference to moral association. In: Eastlake CL, editor. Theory of Colors. 2nd ed. Cambridge, MA: MIT Press; 1840. p. 321-3.

[12]. Carruthers HR, Morris J, Tarrier N, Whorwell PJ. The Manchester Color Wheel: development of a novel way of identifying color choice and its validation in healthy, anxious and depressed individuals. BMC Med Res Methodol. 2010 Feb 9;10:12. Pubmed PMID: 20144203.

[13]. Ekman P, Freisen WV, Ancoli S. Facial signs of emotional experience. Journal of personality and social psychology. 1980 Dec;39(6):1125.

[14]. Cimbalo RS, Beck KL, Sendziak DS. Emotionally toned pictures and color selection for children and college students. The Journal of Genetic Psychology. 1978 Dec 1;133(2):303-4.

[15]. Odom AS, Sholtz SS. The reds, whites, and blues of emotion: Examining color hue effects on mood tones. National Undergraduate Research Clearinghouse. 